\title{
Stability and Electronic Properties of 2D
}

\section{Nanomaterials Conjugated with Pyrazinamide}

\section{Chemotherapeutic - A First Principles Cluster}

\section{Study}

Nabanita Saikia, Maximilian Seel and Ravindra Pandey ${ }^{*}$

Department of Physics, Michigan Technological University, Houghton, Michigan 49931, USA

\section{AUTHOR INFORMATION}

\section{Corresponding Author}

*E-mail: pandey@mtu.edu (R. Pandey).

Telephone: +1-906-487-2086. 
Table S1. Comparison of calculated HOMO-LUMO energy gap (eV), average bond length $(\AA)$, and binding energy $\left(\Delta E_{b}, \mathrm{eV}\right)$ for selected PZA-conjugated clusters of 72 and 120 atoms in aqueous solution.

\begin{tabular}{cccc}
\hline $\begin{array}{c}\text { System } \\
\mathbf{( P B E - 6 - 3 1 G ( d , p ) / D 2 )}\end{array}$ & $\begin{array}{c}\text { Energy Gap } \\
\mathbf{( e V )}\end{array}$ & $\begin{array}{c}\text { Bond length } \\
(\mathbf{\AA})\end{array}$ & $\begin{array}{c}\Delta E_{b} \mathbf{( P Z A / c l u s t e r )} \\
(\mathbf{e V})\end{array}$ \\
\hline 72 atom clusters $\mathbf{( X 5 4 H 1 8 )}$ & & & \\
\hline Graphene & 1.89 & 1.42 & -0.66 \\
BN & 4.69 & 1.45 & -0.82 \\
Silicene & 0.77 & 2.28 & -0.96 \\
SiC & 2.05 & 1.80 & - \\
Phosphorene & 1.86 & 2.26 & -0.61 \\
& & & \\
\hline 120 atom cluster (X96H24) & & & -0.68 \\
\hline Graphene & 1.34 & 1.42 & -0.84 \\
BN & 4.49 & 1.45 & - \\
Silicene & 0.53 & 2.28 & - \\
SiC & 1.65 & 1.80 & -0.66 \\
Phosphorene & 1.52 & 2.26 & \\
\hline
\end{tabular}


Table S2. Calculated structural properties of PZA molecule in the solvated phase obtained at the level of DFT (PBE)-D2 theory. Bond lengths are given in Å. Bond angles and dihedral angles are given in degrees.

\begin{tabular}{|c|c|c|c|}
\hline \multirow{2}{*}{$\begin{array}{l}\text { Structural } \\
\text { parameters }\end{array}$} & \multirow[t]{2}{*}{ Experiment $^{1}$} & \multicolumn{2}{|c|}{ Theory } \\
\hline & & This work & Reference $^{2}$ \\
\hline \multicolumn{4}{|l|}{ Bond lengths } \\
\hline $\mathrm{C} 3-\mathrm{C} 2$ & 1.383 & 1.409 & 1.402 \\
\hline $\mathrm{C} 1-\mathrm{C} 4$ & 1.374 & 1.406 & 1.399 \\
\hline $\mathrm{C} 3-\mathrm{C} 10$ & 1.501 & 1.518 & 1.515 \\
\hline $\mathrm{C} 10-\mathrm{O} 14$ & 1.241 & 1.238 & 1.217 \\
\hline $\mathrm{C} 2-\mathrm{H} 6$ & 1.001 & 1.096 & 1.088 \\
\hline $\mathrm{C} 1-\mathrm{H} 5$ & 1.020 & 1.096 & 1.088 \\
\hline $\mathrm{C} 4-\mathrm{H} 7$ & 0.920 & 1.096 & 1.088 \\
\hline $\mathrm{N} 11-\mathrm{H} 12$ & 0.867 & 1.017 & 1.011 \\
\hline N11 - H13 & 0.871 & 1.016 & 1.012 \\
\hline $\mathrm{N} 11-\mathrm{C} 10$ & 1.309 & 1.365 & 1.377 \\
\hline $\mathrm{N} 8-\mathrm{C} 3$ & 1.344 & 1.351 & 1.341 \\
\hline $\mathrm{N} 8-\mathrm{C} 4$ & 1.355 & 1.343 & 1.333 \\
\hline N9 $-\mathrm{C} 2$ & 1.329 & 1.347 & 1.338 \\
\hline N9-C1 & 1.353 & 1.346 & 1.336 \\
\hline \multicolumn{4}{|l|}{ Bond angles } \\
\hline $\mathrm{C} 4-\mathrm{N} 8-\mathrm{C} 3$ & 115.4 & 115.9 & 116.3 \\
\hline $\mathrm{C} 1-\mathrm{N} 9-\mathrm{C} 2$ & 116.0 & 115.6 & 115.9 \\
\hline $\mathrm{N} 8-\mathrm{C} 3-\mathrm{C} 2$ & 121.9 & 121.5 & 121.3 \\
\hline $\mathrm{N} 9-\mathrm{C} 2-\mathrm{C} 3$ & 122.6 & 122.5 & 122.4 \\
\hline $\mathrm{N} 9-\mathrm{C} 1-\mathrm{C} 4$ & 121.4 & 122.0 & 121.9 \\
\hline $\mathrm{N} 8-\mathrm{C} 4-\mathrm{C} 1$ & 122.4 & 122.3 & 122.2 \\
\hline $\mathrm{C} 3-\mathrm{C} 10-\mathrm{N} 11$ & 117.5 & 114.9 & 114.6 \\
\hline $\mathrm{C} 3-\mathrm{C} 10-\mathrm{O} 14$ & 119.1 & 121.4 & 122.3 \\
\hline $\mathrm{N} 11-\mathrm{C} 10-\mathrm{O} 14$ & 123.2 & 123.6 & 123.1 \\
\hline $\mathrm{N} 9-\mathrm{C} 1-\mathrm{H} 5$ & 117.3 & 117.3 & 117.1 \\
\hline $\mathrm{N} 9-\mathrm{C} 2-\mathrm{H} 6$ & 115.3 & 116.7 & 116.5 \\
\hline $\mathrm{C} 10-\mathrm{N} 11-\mathrm{H} 12$ & 117.2 & 117.7 & 120.3 \\
\hline $\mathrm{C} 10-\mathrm{N} 11-\mathrm{H} 13$ & 121.6 & 121.9 & 119.0 \\
\hline \multicolumn{4}{|l|}{ Dihedral angles } \\
\hline $\mathrm{C} 3-\mathrm{C} 10-\mathrm{N} 11-\mathrm{H} 12$ & 177.1 & 176.9 & 173.5 \\
\hline $\mathrm{C} 3-\mathrm{C} 10-\mathrm{N} 11-\mathrm{H} 13$ & 20.5 & 19.7 & 25.6 \\
\hline
\end{tabular}

1. Y. Takaki, Y. Sasada, T.Watanabe, The Crystal Structure of a-Pyrazinamide, Acta Cryst., 1960, 13, 693.

2. V. Chiş, A. Pîrnâu, T. Jurcâ, M. Vasilescu, S. Simon, O. Cozar, L. David, Experimental and DFT study of pyrazinamide, Chem. Phys., 2005, 316, 153. 
Table S3: Comparison of nearest-neighbor distance and binding energies $\left(\Delta E_{b}, \mathrm{eV}\right)$ for the gas and solvated (PCM) phases of the PZA/graphene and PZA/BN 120 atom clusters.

\begin{tabular}{lll}
\hline System & $\begin{array}{c}\text { Nearest-neighbor distance } \\
(d, \AA)\end{array}$ & $\Delta E_{b}(\mathrm{eV})$ \\
\hline PZA/graphene & & \\
\hline Gas phase & 3.16 & -0.76 \\
Solvated & 3.13 & -0.68 \\
\hline PZA/BN & & \\
\hline Gas phase & 3.09 & -0.93 \\
Solvated & 3.02 & -0.84 \\
\hline
\end{tabular}
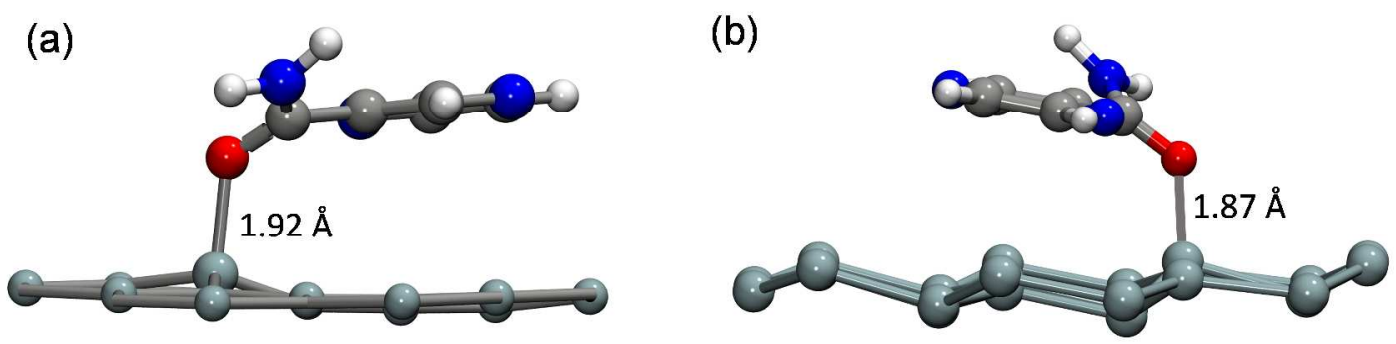

Figure S1. Side views of PZA adsorbed onto (a) $\mathrm{SiC}$ and (b) silicene clusters. 


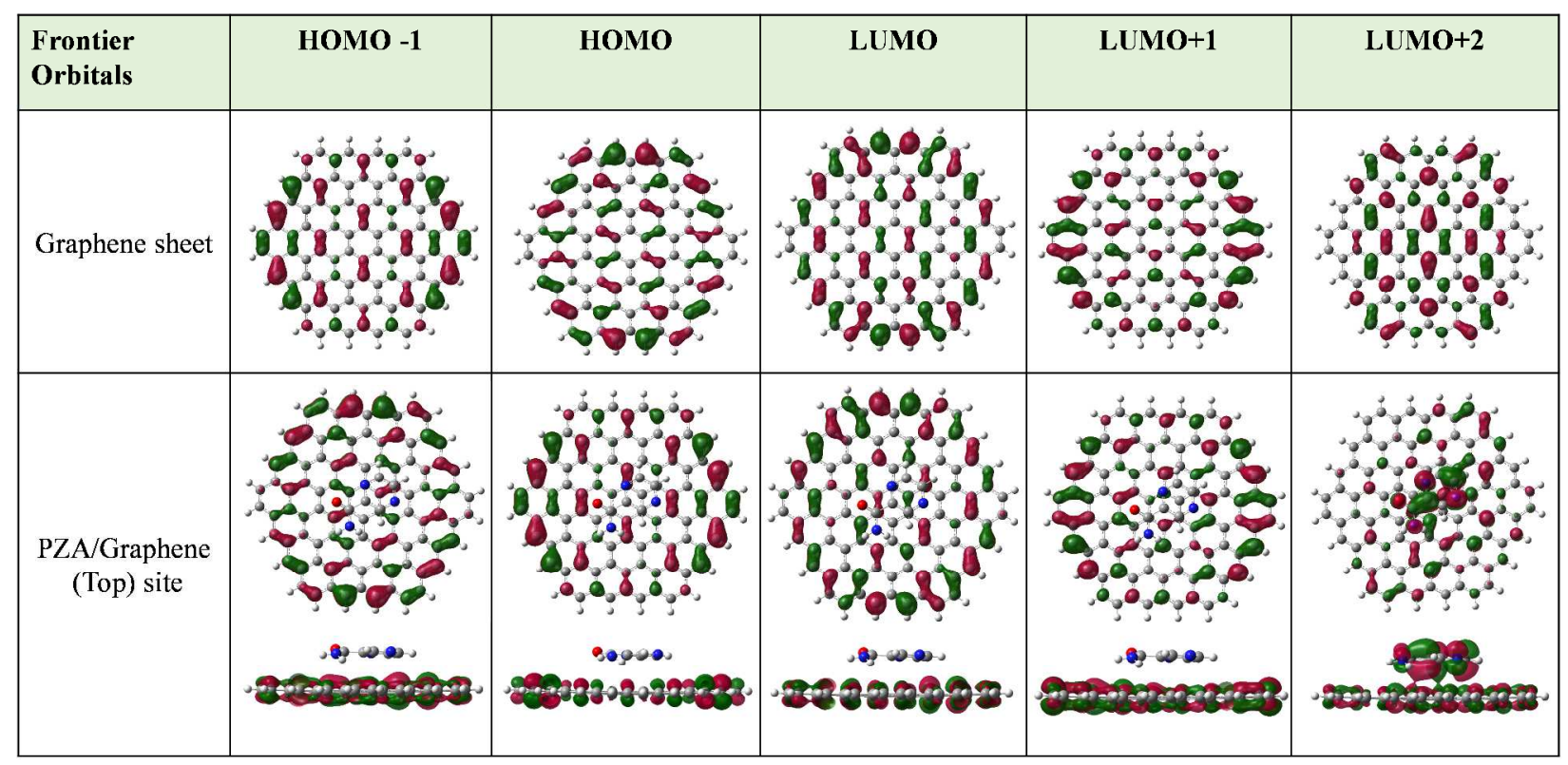

Figure S2. Frontier orbitals corresponding to HOMO - 1, HOMO, LUMO, LUMO +1 and LUMO +2 states of pristine Graphene and PZA/Graphene bioconjugates (Isovalue $=0.02$ arb. Units).

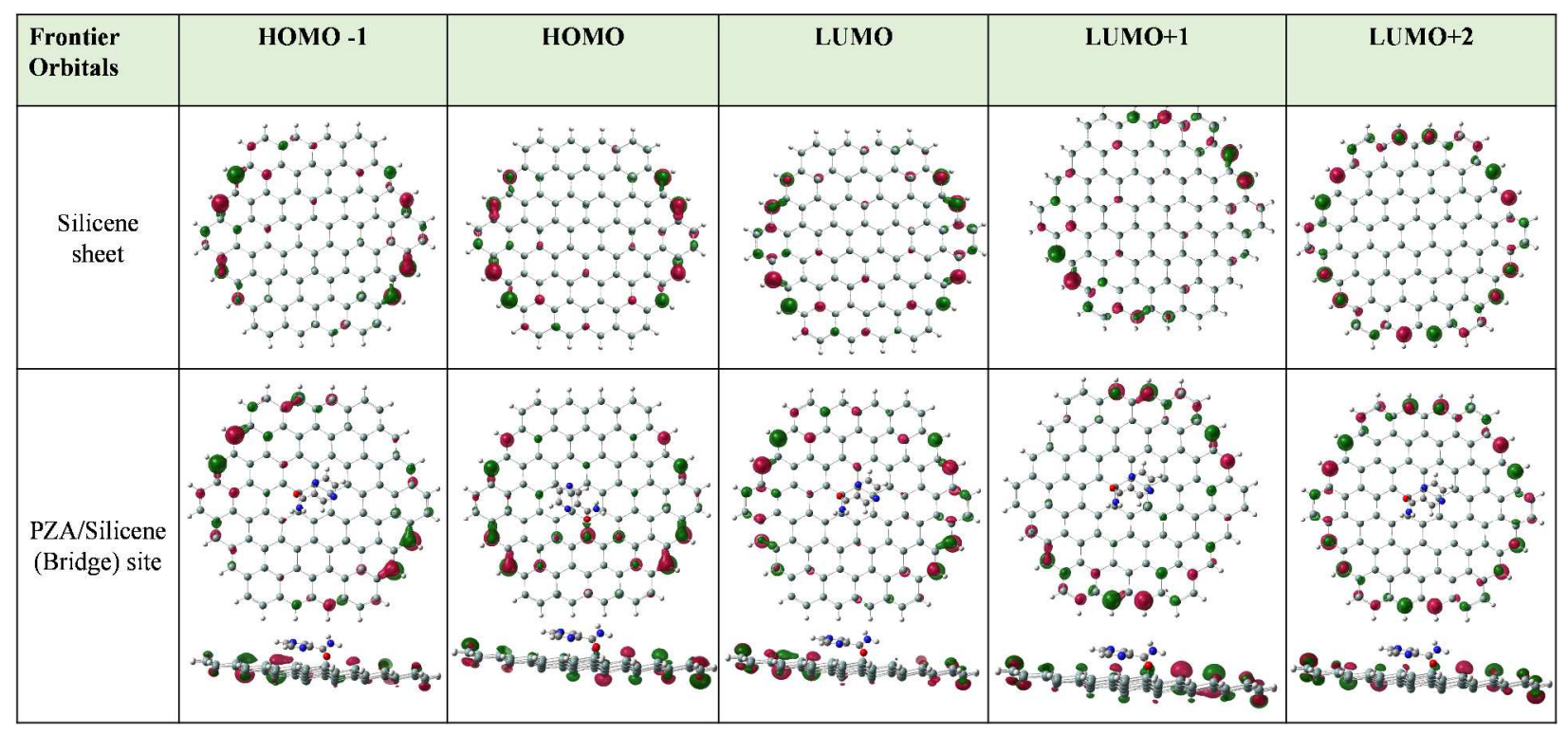

Figure S3. Frontier orbitals corresponding to HOMO - 1, HOMO, LUMO, LUMO +1 and LUMO +2 states of pristine Silicene and PZA/Silicene bioconjugates (Isovalue $=0.02$ arb. Units). 


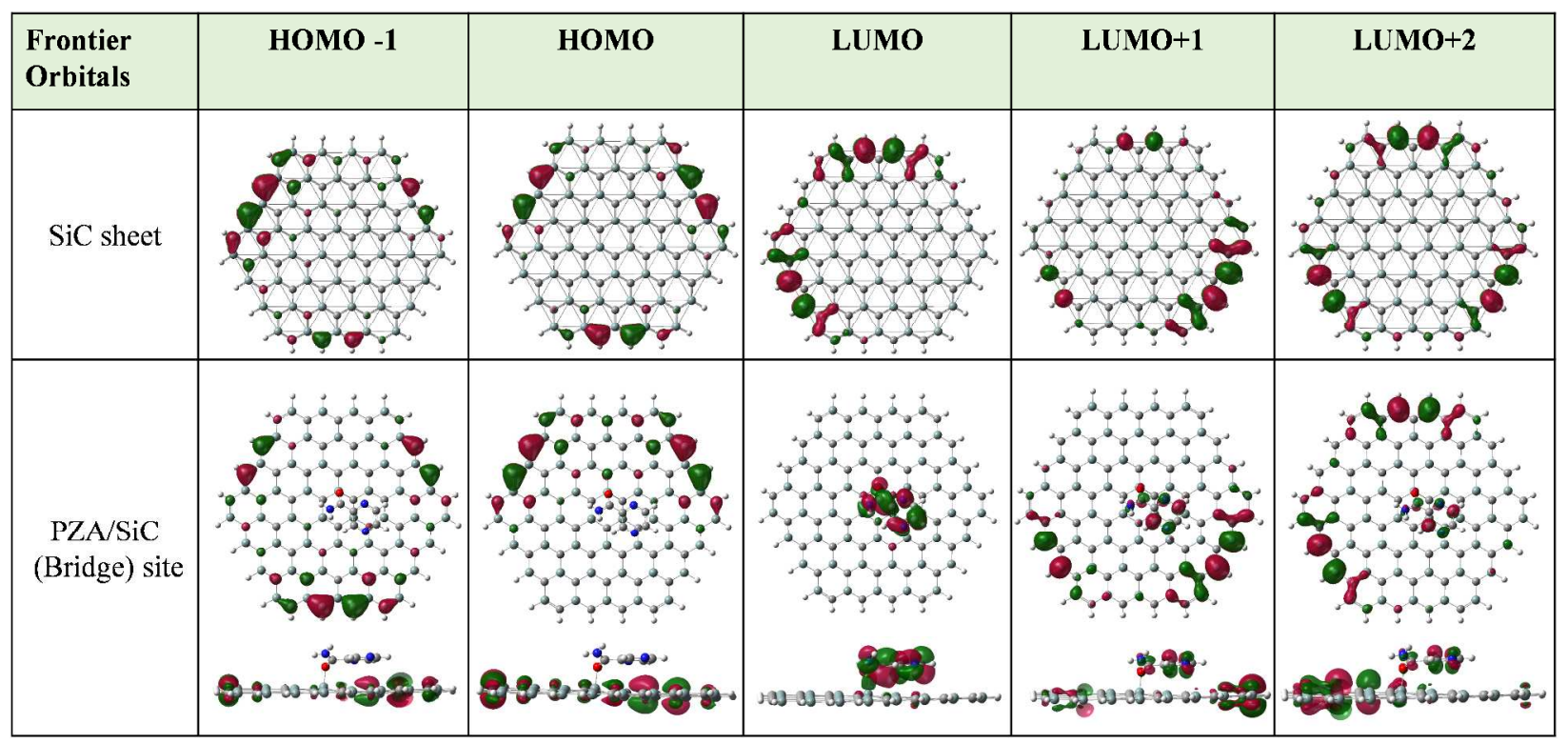

Figure S4. Frontier orbitals corresponding to HOMO - 1, HOMO, LUMO, LUMO +1 and $\mathrm{LUMO}+2$ states of pristine $\mathrm{SiC}$ and $\mathrm{PZA} / \mathrm{SiC}$ bioconjugates (Isovalue $=0.02 \mathrm{arb}$. Units).

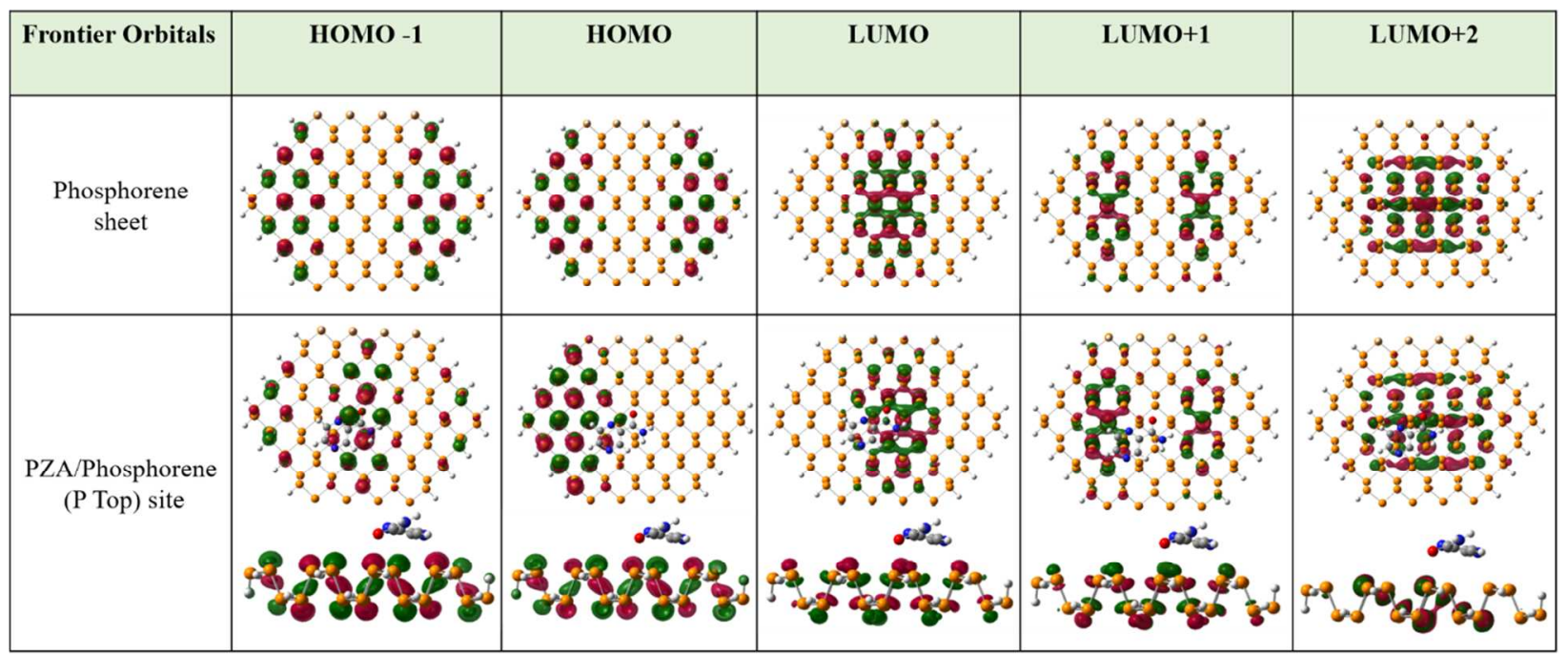

Figure S5. Frontier orbitals corresponding to HOMO - 1, HOMO, LUMO, LUMO +1 and LUMO +2 states of pristine Phosphorene and PZA/Phosphorene bioconjugates (Isovalue $=0.02$ arb. Units). 